\title{
The oesophageal diverticulum of Dirioxa pornia studied through micro-CT scan, dissection and SEM studies
}

\author{
Kala Bhandari ${ }^{1}$, Peter Crisp $^{2^{*}}$ and Michael A. Keller ${ }^{1^{*}}$
}

\begin{abstract}
Background: Dirioxa pornia (Diptera, Tephritidae) (Island fly) is an Australian native species related to a number of pestiferous fruit flies but, unlike many of the pest species, has not been studied extensively due to its non-pest status. However, due to D. pornia's apparent reliance on the bacteria for survival it is an ideal species to undertake studies into interaction between Tephritid species and bacteria associated with the intestinal tract. The oesophageal diverticulum, which is a blind-ended protrusion of the oesophagus, has been studied, described and characterised in many other Tephritid species. Unlike many other species where the oesophageal diverticulum has been observed the organ was only observed in male D. pornia. It is speculated that this sexual dimorphism the organ may be the primary location to host beneficial bacteria in the involved in the production of the nuptial gift and the mating success of this Tephritid species. In case of D. pornia, however, no study on any area of the digestive system has been conducted. This study was conducted to locate and characterize the oesophageal diverticulum in D. pornia. A virtual dissection of the alimentary tract was made through micro-computer tomography studies. These studies were followed by dissection and scanning microscopy studies to elucidate the presence of bacteria.

Results: The oesophageal diverticulum of D. pornia is part of the foregut and distends from the oesophagus within the head of the fly. The shape of the oesophageal diverticulum corresponds with the Ceratitis type. Scanning microscopy studies of the oesophageal diverticulum show rod-shaped bacterial cells residing along with yeast cells in the lumen. The organ was only observed in male specimens.

Conclusions: This study classifies the oesophageal diverticulum of D. pornia under the "Ceratitis type" of oesophageal diverticula in Tephritid species. The study also proves that micro-CT scanning is possible to locate soft tissues in Tephritid species and the Avizo ${ }^{\oplus}$ Fire software can be successfully used to visualize 3 dimensional (3D) images from $x$-rays. The methods used in this experiment can be used in future studies for visualising soft tissues of adult Tephritid species through micro tomography. There is sexual dimorphism with the organ only found in males. Finally this study shows that bacteria are present in the oesophageal diverticulum of D. pornia.
\end{abstract}

Keywords: Island fly, Tephritidae, 3D micro-imaging, Avizo ${ }^{\oplus}$ fire, Bacteria

\section{Background}

Fruit flies (Diptera; Tephritidae) constitute some of the major pests of horticultural crops. Control measures such as the sterile insect technique (SIT) are used to keep populations of many fruit fly species within manageable limits. In Australia SIT is used both as management

\footnotetext{
* Correspondence: Peter.crisp@sa.gov.au; Mike.Keller@adelaide.edu.au ${ }^{2}$ Entomology Unit, South Australian Research and Development Institute, PIRSA, Adelaide, SA, Australia

'School of Agriculture, Food and Wine, The University of Adelaide, Adelaide, SA, Australia
}

and eradication tool for the Bactrocera tryoni (Froggatt) (Queensland fly) and Ceratitis capitata (Wiedemann) (Mediterranean fly). To improve the efficacy of a new facility for the production of B. tryoni, for use in SIT programs, new rearing methods and diets are being developed. There are various published studies dedicated to the enhancement of SIT practices, including the potential of the exploitation of gut bacteria to improve on rearing efficacy and fly fitness [1-3].

Insects have been shown to have a range of complex relationships with bacteria varying from casual interactions 
to complete dependence $[4,5]$. Non-pathogenic mutualistic relationships between insects and their gut bacteria are reported to mainly involve nutritional interactions [6-8]. In some cases the relationship is so critical that when the bacteria are eliminated from their host, the host cannot survive on its natural food source alone [9].

Bactrocera oleae (Rossi) (Olive fly) is the first example of a Tephritid known to host symbiotic bacteria. Petri, in as early as 1909 , hypothesised that the $B$. oleae gutsymbionts multiply in the oesophageal diverticulum (oesophageal bulb/ pharyngeal vessel) and are released into the gut to be digested by the fly [10]. The oesophageal diverticulum was considered exclusive to the Olive fly until 1973, when Girolami discovered and described the organ in most Tephritid species [11], as follows,

a. The 'Dacus' type. The Dacus type is the largest of the oesophageal diverticula and the bacteria multiply near the elongated basal epithelial cells.

b. The 'Ceratitis' type. This type is present in Trypetinae and Dacinae, except B. oleae. The bacteria, which are easily visible in Trypetinae, multiply in the lumen.

c. The 'Ensina' type. This is egg-shaped and present in Tephritinae, except Terellini. Bacteria are not known to be present in this oesophageal diverticulum.

d. The 'Chaetorellia' type. This is present in the tribe Terellini and the features combine some of the characteristics of the Ceratitis and Ensina types.

The oesophageal diverticulum has also been studied in the Apple maggot, Rhagoletis pomonella (Walsh) $[12,13]$. The studies hypothesise that the oesophageal diverticulum is present in order to house symbiotic bacteria which are released into the gut lumen as and when required by the host insect. This hypothesis is similar to those made by Petri and Girolami $[10,11]$.

Dirioxa pornia (Walker) (Tephritidae: Phytalmiinae) is a fruit fly native to Australia and New Caledonia that lays its eggs in damaged fruit [14]. However, a small number of cases of $D$. pornia larvae being found in exported citrus have been reported from overseas markets which lead to initial laboratory studies in South Australia. In countries such as Thailand and New Zealand it is listed as a quarantine pest. It was found that unlike other fruit fly species (such as C. capitata and B. tryoni), D. pornia cultures could only be maintained when their diets were supplemented with artificially grown gut-bacteria [15]. There have been only limited studies on this species $[16,17]$. The study on the structure of the alimentary tract, including the oesophageal diverticulum has not been made. Elucidating the relationship between the oesophageal diverticulum and bacteria in this species may provide a better understanding of the importance of bacteria in the biology of Tephritid species in general, which in turn could be used in improving efficiency in SIT mass rearing facilities.

Earlier in this study the precise location of the oesophageal diverticulum could not be established through dissection methods alone. To accurately elucidate the location of the oesophageal diverticulum micro-computed tomography (micro-CT) scan of the head of adult D. pornia was conducted. Micro-CT is an emerging technology for the imagery of insects $[18,19]$. An advantage that the micro-CT technique has over classical dissection methods is that it is non-destructive and is precise in terms of organ location. Additionally, a single scan can be saved digitally instead of having to physically preserve samples in chemical substances that may either be toxic or can deteriorate over time. Mainly, this technique was selected in order to develop methods to conduct micro-CT scans on Tephritid species.

This study will provide a clear understanding of the structure and location of the oesophageal diverticulum of $D$. pornia and contribute to the development of micro-computed tomography as a non-destructive dissection method to study the structure within Tephritidae and other insects. It also aims to elucidate the presence of bacterial cells within the oesophageal diverticulum of the $D$. pornia through scanning electron micrographs. This information will contribute to the understanding of the relationship between Tephritid fruit flies and the communities of bacteria in their gut and provide a basis for ongoing research in the field.

\section{Methods}

\section{Island fly culture}

Island flies used for the scans were obtained from cultures maintained at the Waite Insectary, School of Agriculture Food and Wine, University of Adelaide, Urrbrae, South Australia, at $27^{\circ} \mathrm{C}$; RH $70-80 \%$ and L:D 13:11 (natural light supplemented by fluorescent lighting). Flies for the culture were sourced from Waikerie and Mypolonga in South Australia's Riverland, male and female flies were trapped in McPhail traps containing Putrescine (FFP) and ammonium acetate (FFA) lures (Suterra LLC, Bend, OR, USA). Adult flies were provided with a diet of hydrolysed yeast and water ad libitum supplemented with Enterobacter spp isolated from the gut of wild D. pornia collected from Loxton in 2011 [15]. Fresh oranges were poked with the help of a needle and placed in adult cages for oviposition and larval development.

\section{Preliminary dissections}

Prior to CT and SEM scans a series of 100 dissections, 50 male and 50 female, of adult $D$. pornia were conducted 
with the help of a Nikon SMZ25 stereomicroscope. The age of male and females flies dissected in this manner ranged from $24 \mathrm{~h}$ to 3 weeks post eclosion to observe any developmental changes that may have affected outcomes.

\section{Preparation of island flies for CT scans}

Methods for dehydration of the flies were modified from Alba-Trecedor [20]. Our method differs from his mainly in the fixing and dehydration process. In our case, when the samples were fixed with $4 \%$ Paraformaldehyde in $\mathrm{PBS}+4 \%$ Sucrose at a $\mathrm{pH}$ of 7.2 prior to dehydration as described in his method, the resulting images appeared unclear. This may have occurred due to fluids trapped during the fixing process. Therefore we did not fix the sample. Instead we directly dehydrated the sample in a graded series of ethanol. Dehydrating of the sample at $70 \%$ which was the method used by Alba-Trecedor also resulted unclear images and tissue damage. In order to avoid this we dehydrated the specimen using a graded series of ethanol at 30, 50, 70, 80, 90 and 100\% which showed better results. Similarly, staining the sample with $1 \%$ Iodine for more than $6 \mathrm{~h}$ showed better contrast of the softer tissues in the CT scans than staining them with $1 \%$ Iodine for $3 \mathrm{~h}$ only.

For this study, 5 day-old male flies were selected from the culture and euthanized at $-18{ }^{\circ} \mathrm{C}$ for $3 \mathrm{~min}$. Euthanized flies were dehydrated in a graded series of ethanol starting at 30, 50, 70, 80, 90 and $100 \%$ for 30 min each. The dehydrated flies were then stained with $1 \%$ iodine in $100 \%$ ethanol for over $6 \mathrm{~h}$. The stained flies were then critically dehydrated in hexamethyldisilazane (HMDS) for $2 \mathrm{~h}$ with one fresh change of HMDS in between. They were left to dry overnight under a fume hood. One fly per scan was selected and then mounted over the top of the axis of a micro-CT scanner (SkyScan 1072, Bruker microCT, Belgium) by sticking it with Araldite $^{\circ}$ glue (Selleys ${ }^{\oplus}$, Padstow, New South Wales, Australia) and left to dry for 20-30 min.

\section{Micro-CT scanning in SkyScan 1072}

The micro-CT scan and analysis of the head of D. pornia was performed at Adelaide Microscopy (Medical School North, Frome Road, The University of Adelaide, SA, Australia) with a Skycan 1072 (Bruker microCT, Kontich, Belgium). The specifications used were; beam energy set at $23 \mathrm{kV}$, current set at $120 \mu \mathrm{A}$, cross-section pixel size set to of $3.67 \mu$; exposure set at $3.4 \mathrm{~s}$ and rotation set to $180^{\circ}$ with images captured every $0.225^{\circ}$.

\section{Post-scan image processing}

Scanned images that were saved in tagged image file format (TIFF) were reconstructed with the help of the NRecon software (Bruker microCT, Kontich, Belgium). The region of interest (ROI) was identified and the dynamic range selected, following which the image was adjusted for misalignment compensation and in this instance fine tuning/ beam hardening was performed. The image data were then converted into bitmap (BMP) format. The new reconstructed image data were then used to segment, recolour and visualise the alimentary tract of the fly in 3D with the help of the Avizo ${ }^{\circ}$ Fire 8.1 software (ThermoFisher Scientific Hillsboro, Oregon, USA). A volume rendering module was attached to the data file and the alpha scale adjusted in order to reveal a semitransparent scanned image of the fly. This was followed by the creation of a label field. An interactive segmentation was performed for the various structures and organs of the scanned 3D image. After the segmentation was complete the new surface generated was visualised with the help of the surface-view and volume rendering modules. Images were captured and saves as TIFF files.

\section{Dissection of $D$. pornia to study the alimentary tract}

Seven $24 \mathrm{~h}$-old adult males were removed from the culture and provided with dyed sucrose solution (Queen Rainbow Food Colours, Australia) for $12 \mathrm{~h}$. One adult was selected and dissected with the help of a Nikon SMZ25 stereomicroscope. The dye helped locate the alimentary tract during dissection. Images of the dissected areas were captured with the help of the attached camera.

\section{Scanning electron microscopy studies of the oesophageal diverticulum of $D$. pornia}

For the SEM studies, 8 adult male $D$. pornia collected from an orchard at Waikerie, SA, were selected. The flies were caught on the leaves and immediately chilled to transportation and processing. The oesophageal diverticuli were removed and fixed in in $4 \%$ Paraformaldehyde in PBS, $4 \%$ sucrose at a pH of 7.2 for $30 \mathrm{~min}$. 2-3 washes were made in PBS $+4 \%$ sucrose for 5 min each. The washed oesophageal diverticuli were then post-fixed in $2 \% \mathrm{OsO}_{4}$ in $\mathrm{PBS}$ for $1 \mathrm{~h}$. They were then dehydrated in a graded series of ethanol at 70, 90 and $100 \%$ with 2 changes per concentration of ethanol at a 15 min interval, with an additional third change made for the last concentration of $100 \%$ ethanol. This was followed by critical point dehydration in a Bal-Tec CPD 030 Critical Point Dryer. The dried oesophageal diverticuli were then mounted on SEM stubs and coated with platinum. They were observed under a Philips XL20 Scanning electron microscope set at beam energy of $10 \mathrm{keV}$ the next day. Images were captured with the help of the CCD camera and were saved in TIFF format.

\section{Results}

\section{Preliminary dissections}

During preliminary dissections made on the D. pornia adults, it was noted that no female flies of the 50 
dissected had the oesophageal diverticulum, but 48 of the 50 males dissected were seen to have them. The absence of detection of the diverticulum on 2 males may have been the result of the dissection technique or other handling errors. The age of male and females flies dissected in this manner ranged from $24 \mathrm{~h}$ to 3 weeks post eclosion, the 2 males were the Oesophageal Diverticulum was not detected were not of a single age cohort. This finding has led to another study on the sexual dimorphism in $D$. pornia, which will be published in future. Further as part of the scanning technique optimisation 5 male and 2 female flies were prepared, scanned and the images at least partially processed, results presented here are from one male but were typical of all males studied, no diverticulum was detected in either female scanned.

\section{Micro-CT images}

Successful 3D micrographs of the oesophageal diverticulum of $D$. pornia were developed with the help of the Avizo $^{\circ}$ Fire 8.1 software. The oesophageal diverticulum was coloured green and the other regions of the alimentary tract coloured orange. To visualize the position of the oesophageal diverticulum in relation to other organs, the alpha scale (a toggle feature found in the Volume
Rendering module under Project View of the software application) was manipulated when the image was viewed from different angles. This helped in marking the precise location of the oesophageal diverticulum. The oesophageal diverticulum is located in the anterior area of the head of the adult $D$. pornia. It is positioned proximal to the left hemisphere of the brain by branching out from the oesophagus (Figs. 1 \& 2).

\section{Dissection of the alimentary tract of $D$. pornia}

With the help of the new found location of the oesophageal diverticulum, it was possible to successfully dissect the entire alimentary tract including the oesophageal diverticulum from the cephalic area. The stomodaeum or the foregut consists of the labella, pharynx, oesophagus, oesophageal diverticulum, crop and cardia. The mesenteron extends from the cardia up to the Malpighian tubules, and the proctodeum continues from the pylorus and Malpighian tubules through the ilium, colon and up to the rectum (Fig. 3).

\section{SEM studies of the oesophageal diverticulum}

SEM images of the oesophageal diverticulum reveal a clear visual of the bulb-shaped oesophageal diverticulum (Fig. 4a.). Slicing the bulb with the help of a scalpel blade reveals rod-shaped bacterial mass filling the lumen. a

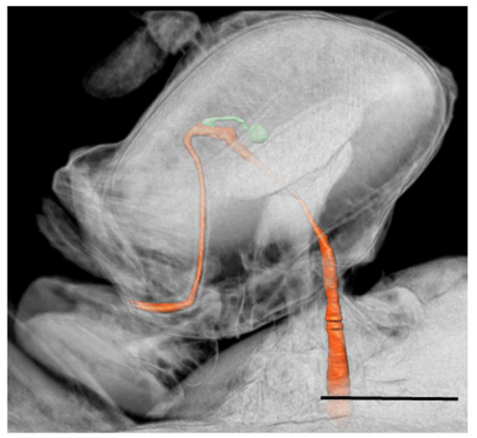

c

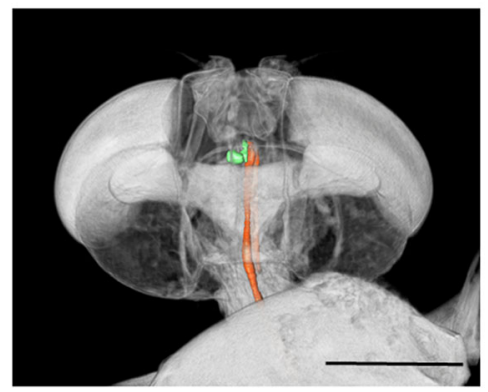

b

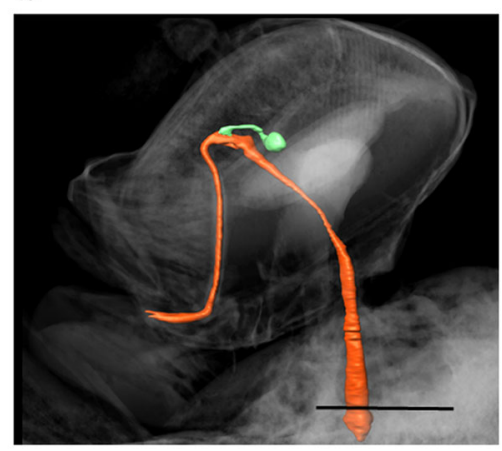

d

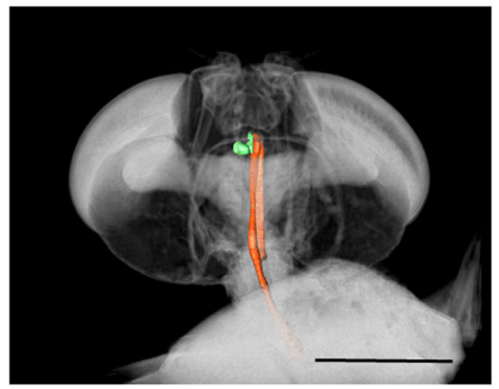

Fig. $1 \mathrm{~A}$ three dimensional reconstruction of the section of the alimentary tract within the cephalic region of Dirioxa pornia. Lateral view (a, b); dorsal view $(\mathbf{c}, \mathbf{d})$. The oesophageal diverticulum is highlighted in green and the rest of the alimentary tract in orange. The alpha scale manipulated between $\mathbf{a}(0.0279) \& \mathbf{b}(0.0079)$ and $\mathbf{c}(0.0489) \& \mathbf{d}(0.0189)$ using the Avizo ${ }^{\circledast}$ Fire 8.1 software edition to compare the location of the oesophageal diverticulum against surrounding tissues and organs. $\mu \mathrm{CT}$ Scan performed in SkyScan 1072 set at $24 \mathrm{keV}, 120 \mu \mathrm{A}$, image resolution of $3.4 \mu$; images captured at $180^{\circ}$ rotation of $0.225^{\circ}$ steps each. Scale bar $=0.5 \mathrm{~mm}$ 


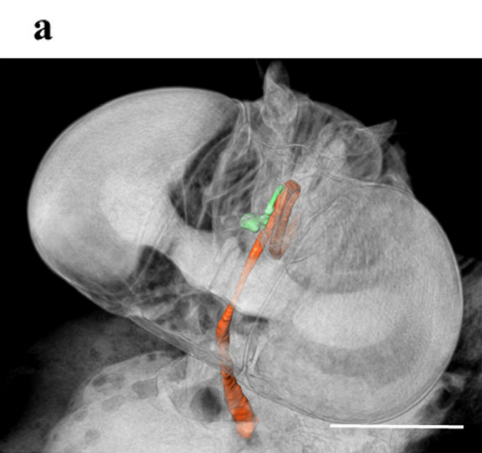

c

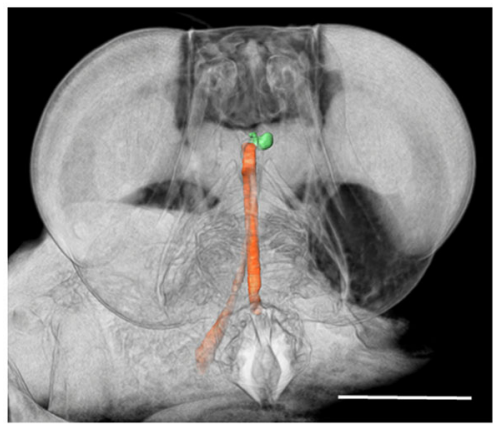

b

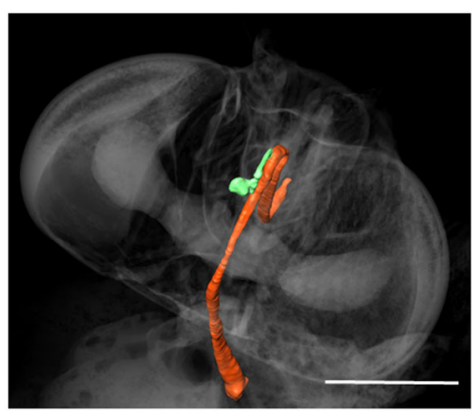

d

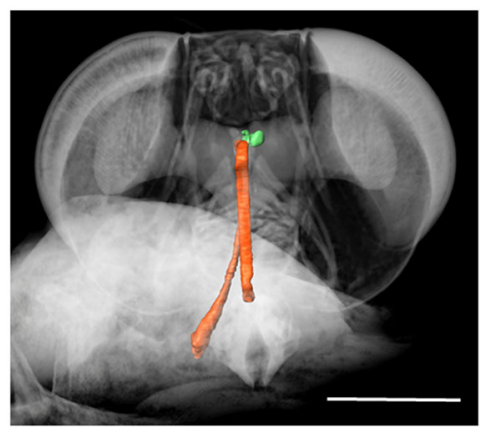

Fig. 2 A three dimensional reconstruction of the section of the alimentary tract within the cephalic region of Dirioxa pornia. Posterior-superior view $(\mathbf{a}, \mathbf{b})$; anterior view $(\mathbf{c}, \mathbf{d})$. The oesophageal diverticulum is highlighted in green and the rest of the alimentary tract in orange. The alpha scale has been manipulated between $\mathbf{a}(0.0339) \& \mathbf{b}(0.0089)$, and $\mathbf{c}(0.0339)$ \& $\mathbf{d}(0.0139)$ using the Avizo ${ }^{\otimes}$ Fire 8.1 software edition to compare the location of the oesophageal diverticulum against surrounding tissues and organs. $\mu \mathrm{CT}$ Scan performed in SkyScan 1072 set at $24 \mathrm{keV}$, $120 \mu \mathrm{A}$, image resolution of $3.4 \mu$; images captured at $180^{\circ}$ rotation of $0.225^{\circ}$ steps each. Scale bar $=0.5 \mathrm{~mm}$

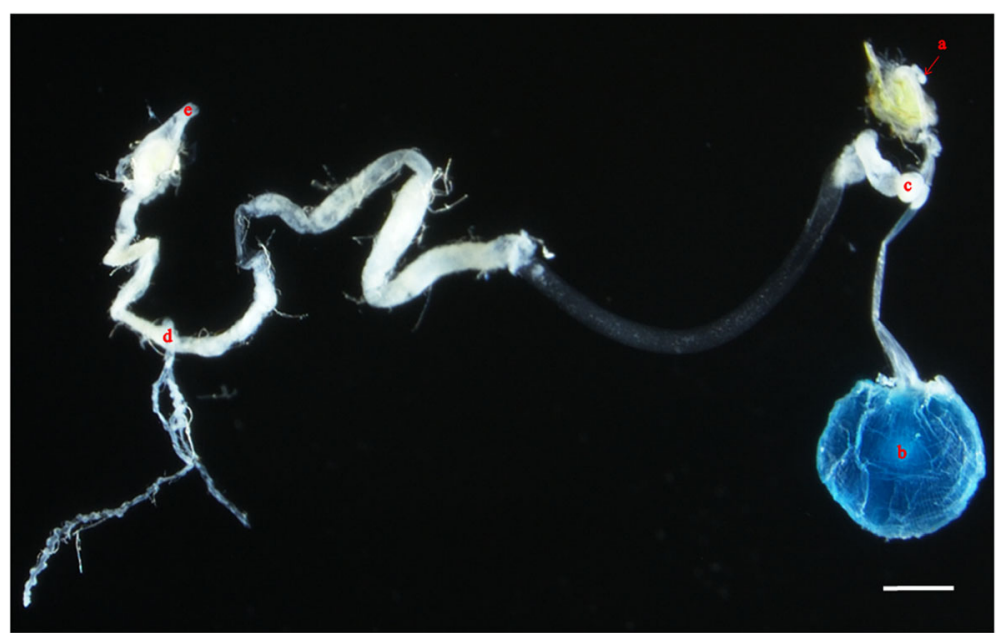

Fig. 3 A photographic image of the dissected alimentary tract of D. pornia (viewed with a Nikon SMZ25 microscope). The crop is filled with blue dyed sugar solution fed to the fly prior to dissection (for contrast). $\mathbf{a}$; oesophageal diverticulum, $\mathbf{b}$; crop, $\mathbf{c}$; cardia, $\mathbf{d}$; Malpighian tubule, e; rectum. Stomodaeum (a-c), mesenteron $(\mathbf{c}-\mathbf{d})$, proctodeum $(\mathbf{d}-\mathbf{e})$. Scale bar $=0.5 \mathrm{~mm}$ 


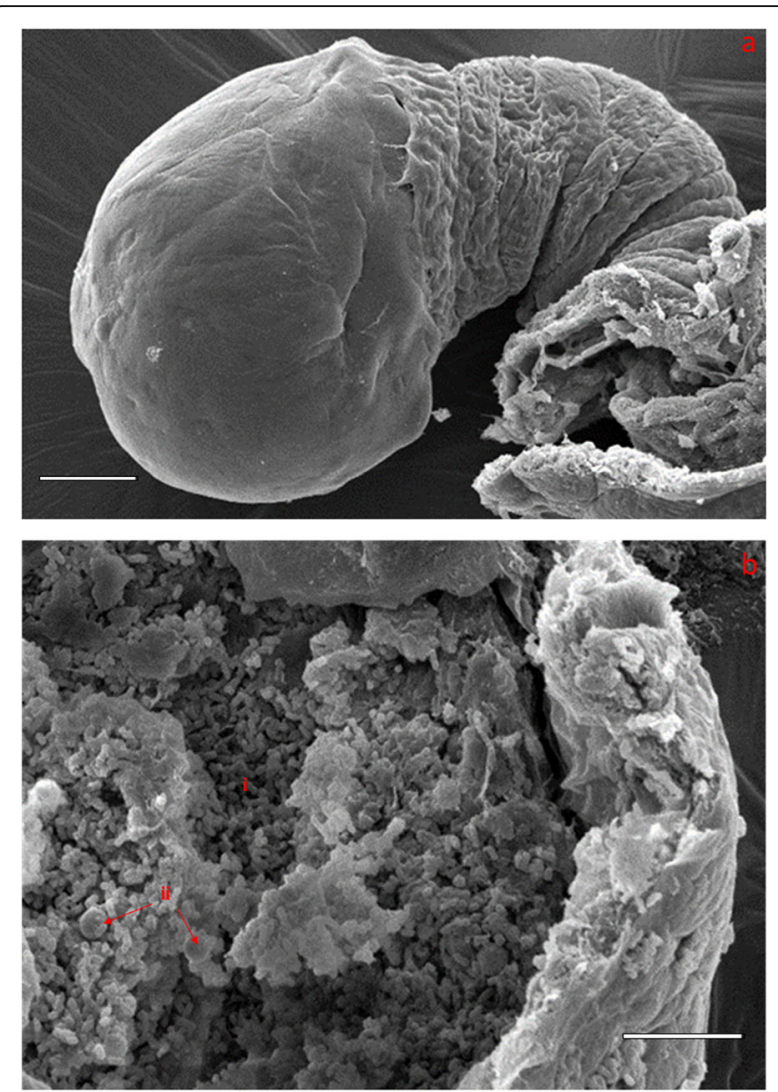

Fig. 4 a. A SEM micrograph of the oesophageal diverticulum of $D$. pornia. The shape corresponds to the 'Ceratitis' type. Scale bar $=20 \mu$. b. An opened oesophageal diverticulum of $D$. pornia showing rodshaped bacterial mass filling the lumen (i). Yeast cells (ii) are also visible. SEM used, Philips XL20, Beam energy set at $10 \mathrm{keV}$. Scale bar $=10 \mu$

Isolated yeast cells are also visible alongside the bacterial cells (Fig. 4b).

\section{Discussion}

In our studies, the dissection of the complete alimentary tract (including the oesophageal diverticulum) of the adult $D$. pornia, were more precise after obtaining results of the micro-CT scan. This is because of the newfound knowledge of the precise location of the oesophageal diverticulum, which helped to be extra careful during dissections. The tissues connecting the foregut to the oesophageal diverticulum can be easily tornapart because of which the oesophageal diverticulum was not found in many previous dissections. The alimentary tract of the adult male $D$. pornia does not have any remarkable difference from that of C. capitata [21]. The scanning electron micrographs of the oesophageal diverticulum of $D$. pornia reveal that the oesophageal diverticulum corresponds to the Ceratitis type under the classification system of oesophageal diverticula [11]. Besides the shape, the bacterial mass that fills the lumen of the oesophageal diverticula also correspond with that of C. capitata [21].

The presence of uniform rod shaped bacteria inside the lumen of the oesophageal diverticula vaguely suggests that there may be a group of bacterial species that reside or enter the oesophageal diverticulum like that of C. capitata and B. oleae $[21,22]$. In the case of studies that were carried out on $C$. capitata, the most dominant bacterial species found in the oesophageal diverticulum were Klebsiella oxytoca and Pantoea agglomerans [21]. In the case of B. oleae, the species Ca. Erwinia dacicola, has been found to be the major symbiont of the fly and present in the oesophageal diverticulum [23-26, and]. This species is non-culturable whereas the two predominant bacterial species founds in C. capitata are. Structurally, however, they are all rod-shaped and between the size of $1-5 \mu \mathrm{m}$. The outcome of those studies indicate that any number of and any kind of bacterial species could be present in the oesophageal diverticulum of $D$. pornia. Culture dependent as well as culture independent studies, not reported here, were carried out to further identify, quantify as well as characterize the microbial community seen through the SEM studies on $D$. pornia.

The presence of yeast cells in the oesophageal diverticulum indicate that $D$. pornia either ingest them or they are inherited and that they may play some part in the digestive role of the fly. A recent study on yeast in Bacterocera tryoni (Froggatt) larvae suggests that a diverse group of yeasts are found in the alimentary tract [27]. The study was not clear on whether the yeasts found in the larvae were vertically transmitted, but it is suggested that the yeast is ingested by the larvae. It is evident from the diets that are provided to most laboratory reared Tephritids, that yeast play an important role in the nutrition of the fly. A further study on the role of yeast cells in $D$. pornia needs to be carried out as well.

An older study on the mating behaviour of $D$. pornia hypothesised that the nuptial gift provided by the male is produced in the salivary glands [16]. However, this is not proven with our new found understanding of the sexual dimorphism of the oesophageal diverticulum in adult $D$. pornia, we could add to the hypothesis that perhaps the oesophageal diverticulum of the male plays a role in providing/ supplying important bacteria for successful reproduction of the species. Two unique traits that $D$. pornia have that perhaps most other Tephritids do not, are a. sexual dimorphism with regard to the oesophageal diverticulum and b. the nuptial gift. We could speculate that these differences are connected to each other. A study to prove this hypothesis, however, needs to be carried out.

While our study has mainly focused on the location of the oesophageal diverticulum, its general structure and 
contents with respect to its relationship to symbiotic bacteria, we have not overlooked the potential importance of the crop. The crop has been studied in a few Tephritid species. It is believed to be a storage organ for food and bacteria [28-30]. The post-feeding bubble expelled by the adult flies contains bacteria that may be important for reproduction [29]. The importance and association of bacteria in the crop of adult $D$. pornia is yet to be studied.

Finally, the primary aim of these studies is to understand the relationship of bacteria with Tephritids and manipulate their symbiosis for the improvement of the current SIT. Recent studies have been made for the improvement of the SIT in B. oleae, Glossina sp (Diptera: Glossinidae), C. capitata and Bactrocera cucurbitae $[2,3,31-34]$. In C. capitata and B. cucurbitae the symbiotic bacteria were provided as supplementary diets for the larvae and found that they contribute towards producing healthier sterile adults [32-34]. Similar studies need to be carried out on B. tryoni. With the help of current and future studies carried out on $D$. pornia we may be able to find symbionts that will ultimately help improve the SIT in B. tryoni.

\section{Conclusion}

D. pornia has a digestive tract similar to that of other Tephritid species such as C. capitata. The oesophageal diverticulum of the adult $D$. pornia is located in the anterior area of the head, proximal to the left hemisphere of the brain and can be classified as 'Ceratitis' type.

Micro-CT scans of soft tissues of Tephritid species can be conducted with a SkyScan 1072. The Avizo Fire software can be successfully used to reconstruct 3D images of the CT scans of soft tissues of Tephritid species. Due to the ability to visualise CT images in 3D format, it allows for a better understanding of the overall morphology of organs such as the oesophageal diverticulum in a sensitive and complex structure. The methods used here have provided a guideline for future CT-based studies of all other Tephritid species.

The oesophageal diverticulum of $D$. pornia hosts a group of morphologically homogeneous rod-shaped bacterial cells and possibly some yeast cells. Recently developed molecular methods of bacterial identification and characterization may provide a greater understanding of the diversity of bacterial populations found within the oesophageal diverticulum of $D$. pornia as the next step in understanding the relationship between these bacteria and the host species. Further studies on the exploitation of the relationship between gut bacteria and fruit flies need to be carried out to successfully incorporate in the improvement of SIT and other fruit fly control techniques.

\section{Abbreviations}

3D: Three dimensional; ROI: Region of interest; SIT: Sterile insect technique; TIFF: Tagged image file format

\section{Acknowledgements}

We would like to thank Mr. Andrew Jessup, formerly of the Department of Primary Industries, NSW, for helping KB in the capacity of her Advisor. Adelaide Microscopy, the University of Adelaide, for the SEM and micro-CT studies. We would like to thank Dr. Marc Jones and Ms. Amy Watson from the School of Earth and Environmental Science, University of Adelaide, for their assistance in post- $C T$ image processing of the Island fly. Also the authors would like to thank the International Atomic Energy Agency for its support through the Coordinated Research Program, in particular Kostas Bourtsis and Carlos Cacerers.

\section{About this supplement}

This article has been published as part of BMC Biotechnology Volume 19 Supplement 2, 2019: Proceedings of an FAO/IAEA Coordinated Research Project on Use of Symbiotic Bacteria to Reduce Mass-rearing Costs and Increase Mating Success in Selected Fruit Pests in Support of SIT Application: biotechnology. The full contents of the supplement are available online at https://

bmcbiotechnol.biomedcentral.com/articles/supplements/volume-19supplement-2.

\section{Authors' contributions}

$\mathrm{KB}$ designed the experiment, conducted the study and reconstructed 3D images from micro-CT X-ray scans. MK and PC participated in all steps of the study as supervisor and co-supervisor to KB respectively. All the authors read and approved the final manuscript.

\section{Funding}

The research was funded by South Australian Research and Development Institute, PIRSA, and the University of Adelaide.

\section{Availability of data and materials}

The data files of SEM and micro-CT scans used in the current study are available from the corresponding authors on reasonable request.

\section{Ethics approval and consent to participate}

Not applicable.

\section{Consent for publication}

Not applicable.

\section{Competing interests}

The authors declare that they have no competing interests.

Published: 18 December 2019

\section{References}

1. Augustinos AA, Kyritsis GA, Papadopoulos NT, Abd-Alla AMM, Cáceres C, Bourtzis K. Exploitation of the Medfly gut microbiota for the enhancement of sterile insect technique: Use of Enterobacter sp in larval diet-based probiotic applications PLoS One 2015, doi:https://doi.org/10.1371/journal. pone.0136459.

2. Yuval B, Ben-Ami E, Behar A, Ben-Yosef M, Jurkevitch E. The Mediterranean fruit fly and its bacteria - potential for improving sterile insect technique operations. J Appl Entomol. 2013;137(s1):39-42.

3. Abd-Alla AMM, Bergoin M, Parker AG, Maniania NK, Vlak JM, Bourtzis K, Boucias DG, Aksoy S: Improving Sterile Insect Technique (SIT) for tsetse flies through research on their symbionts and pathogens. J Invertebr Pathol 2013, 112, Supplement 1(0):S2-S10.

4. Dillon RJ, Dillon VM. The gut bacteria of insects: nonpathogenic interactions, Annl Rev Entomol. 2004:49(1):71-92.

5. Baumann P, Moran NA, Baumann LC. Bacteriocyte-Associated Endosymbionts of Insects. In: Rosenberg E, DeLong EF, Lory S, Stackebrandt E, Thompson F, (eds). The Prokaryotes. Berlin: Springer; 2013. p. 465-96.

6. Paul B, Buchner P. Endosymbiosis Anim Plant Microorganisms. New York: Interscience Publishers; 1965.

7. Douglas E, Angela. Lessons from studying insect symbioses. Cell Host Microbe. 2011;10(4):359-67. 
8. Drew R, Courtice A, Teakle I. Bacteria as a natural source of food for adult fruit flies (Diptera: Tephritidae). Oecologia. 1983;60(3):279-84.

9. Douglas $\mathrm{A}$. The microbial dimension in insect nutritional ecology. Funct Ecol. 2009:23(1):38-47.

10. Petri L. Untersuchung uber die darmbakterien der olivenfliege. Zentralblatt Bakteriol Parasitenkd Infekt Hyg. 1910;26:357-67.

11. Girolami V. Reperti morfo-istologici sulle batterio simbiosi del Dacus oleae Gmelin e di altri ditteri tripetidi, in natura e negli allevamenti su substrati artificiali. Redia. 1973;54:269-94.

12. Ratner SS, Stoffolano JG Jr. Development of the esophageal bulb of the apple maggot, Rhagoletis pomonella (Diptera: Tephritidae): morphological, histological, and histochemical study. Annls Entomol Soc America. 1982;75(5):555-62.

13. Ratner SS, Stoffolano JG. Ultrastructural changes of the esophageal bulb of the adult female apple maggot, Rhagoletis pomonella (Walsh) (Diptera : Tephritidae). Int J Insect Morphol Embryol. 1984;13(3):191-208.

14. Baker G, Crisp P, Mo J, Smallridge C: Managing pests of quarantine concern for citrus market access. SARDI (sustainable systems) Adelaide. In: HAL Final Report. Adelaide; 2011

15. Baker GJ, Crisp P: Market access solutions for Fuller's rose weevil and island fly. In; Final Report to Horticulture Australia Limited 2013: 43.

16. Pritchard G. Laboratory observations on the mating behaviour of the island fruit fly Rioxa pornia (Diptera: Tephritidae). Aust J Entomol. 1967;6(2):127-32.

17. Morrow JL, Frommer M, Shearman DC, Riegler M. The microbiome of fieldcaught and laboratory-adapted Australian tephritid fruit fly species with different host plant use and specialisation. Microb Ecol. 2015;70(2):498-508.

18. Gui J, Zou J, Rong J, Hu Z, Li Y, Xi D, Zheng H: Development and evaluation of a high performance micro-CT system for insect or small animal imaging. In: Bioinformatics and Biomedical Engineering (iCBBE), 2010 4th International Conference 18-20 June 2010. 1-4.

19. Brandt J, Doig G, Tsafnat N. Computational aerodynamic analysis of a microCT based bio-realistic fruit fly wing. PLoS One. 2015;10(5):1-16.

20. Alba-Tercedor J. Study of the anatomy of the common housefly Musca domestica Linnaeus, 1758 (Insecta: Diptera, Muscidae) scanned with the SkyScan 1172 high resolution micro-CT. Bruker Micro-CT Users Meeting. 2013;2013:275-89.

21. Marchini D, Rosetto M, Dallai R, Marri L. Bacteria associated with the oesophageal bulb of the medfly Ceratitis capitata (Diptera:Tephritidae). Curr Microbiol. 2002:44(2):120-4.

22. Poinar GO, Hess RT, Tsitsipis JA. Ultrastructure of the bacterial symbiotes in the pharyngeal diverticulum of Dacus oleae (Gmelin) (Trypetidae: Diptera). Acta Zool. 1975;56(1):77-84.

23. Capuzzo C, Firrao G, Mazzon L, Squartini A. Girolami V: 'Candidatus Erwinia dacicola', a coevolved symbiotic bacterium of the olive fly Bactrocera oleae (Gmelin). Int J Syst Evol Microbiol. 2005;55(Pt 4):1641-7.

24. Sacchetti P, Granchietti A, Landini S, Viti C, Giovannetti L, Belcari A. Relationships between the olive fly and bacteria. J Appl Entomol. 2008; 132(9-10):682-9.

25. Estes A. Life in a fly: the ecology and evolution of the olive fly endosymbiont, Candidatus Erwinia dacicola. PhD Dessertation, University of Arizona; 2009. p. 86-153. https://www.researchgate.net/publication/2 77993113_Life_in_a_fly_the_ecology_and_evolution_of_the_olive_fly_ endosymbiont_Candidatus_Erwinia_dacicola.

26. Estes AM, Hearn DJ, Bronstein JL, Pierson EA. The olive fly endosymbiont, 'Candidatus Erwinia dacicola' switches from an intracellular existence to an extracellular existence during host insect development. Appl Environ Microbiol. 2009;75(22):7097-106.

27. Deutscher AT, Reynolds OL, Chapman TA. Yeast: an overlooked component of Bactrocera tryoni (Diptera: Tephritidae) larval gut microbiota. J Econ Entomol. 2017;110(1):298-300.

28. Lloyd A, Drew R, Teakle D, Hayward A. Bacteria associated with some Dacus species (Diptera: Tephritidae) and their host fruit in Queensland. Aust J Biol Sci. 1986:39(4):361-8.

29. Drew RAl, Lloyd AC. Relationship of fruit flies (Diptera: Tephritidae) and their bacteria to host plants. Ann Entomol Soc Am. 1987:80(5):629-36.

30. Stoffolano JG, Haselton AT. The adult dipteran crop: a unique and overlooked organ. Annu Rev Entomol. 2013;58(1):205-25.

31. Estes AM, Nestel D, Belcari A, Jessup A, Rempoulakis P, Economopoulos AP. A basis for the renewal of sterile insect technique for the olive fly, Bactrocera oleae (Rossi). J Appl Entomol. 2012;136(1-2):1-16.

32. Hamden $\mathrm{H}$, Guerfali MMS, Fadhl S, Saidi M, Chevrier C. Fitness improvement of mass-reared sterile males of Ceratitis capitata (Vienna 8 strain) (Diptera:
Tephritidae) after gut enrichment with probiotics. J Econ Entomol. 2013; 106(2):641-7.

33. Augustinos AA, Kyritsis GA, Papadopoulos NT, Abd-Alla AM, Cáceres C, Bourtzis K. Exploitation of the medfly gut microbiota for the enhancement of sterile insect technique: use of Enterobacter sp. in larval diet-based probiotic applications. PLoS One. 2015;10(9):e0136459.

34. Yao M, Zhang H, Cai P, Gu X, Wang D, Ji Q. Enhanced fitness of a Bactrocera cucurbitae genetic sexing strain based on the addition of gut-isolated probiotics (Enterobacter sp.) to the larval diet. Entomol Exp Appl. 2017; 162(2):197-203.

\section{Publisher's Note}

Springer Nature remains neutral with regard to jurisdictional claims in published maps and institutional affiliations.
Ready to submit your research? Choose BMC and benefit from:

- fast, convenient online submission

- thorough peer review by experienced researchers in your field

- rapid publication on acceptance

- support for research data, including large and complex data types

- gold Open Access which fosters wider collaboration and increased citations

- maximum visibility for your research: over $100 \mathrm{M}$ website views per year

At $\mathrm{BMC}$, research is always in progress.

Learn more biomedcentral.com/submissions 